ISSN 0001-6002/2002/44/2/62-67

A cta M édica Costarricense, $@ 2002$

Colegio de M édicos y Cirujanos

\title{
Originales
}

\section{Bloqueo femoral tres en uno para cirugía de fractura transtoncantérica de cadera: seguridad y analgesia}

\author{
Walter Rojas-Rivera ${ }^{1}$, Mohammand Abed-R ${ }^{2}$
}

Objetivo: Determinar la eficacia del bloqueo femoral tres en uno, como técnica anestésica alternativa en la realización de cirugía de cadera, en pacientes A SA III y IV , así como la valoración postquirurgica de la analgesia.

Metodos: se realizó un estudio prospectivo en 12 pacientes, los cuales fueron sometidos a cirugía de cadera, osteosíntesis con pin placa en fractura transtocantérica de fémur, y cuyo estado general fuera clasificado como A SA III o IV. Se le aplicó bloqueo femoral tres en uno y fémoro cutáneo lateral ipsilateral. Se utilizó buvicaína $0.25 \%$, lidocaína $2 \%$, llevada a $40 \mathrm{ml}$ de volumen.

Resultados: la muestra incluyó siete varones y cinco mujeres. La edad media fue de 68.3 años, con límites entre 37 y 93 años. Los pacientes mostraron asociados diagnósticos de hipertensión arterial, diabetes mellitus, asma, cardiopatía isquémica, disrritmias, encefalopatía.

Conclusiones: La estabilidad hemodinámica observada fue excelente, así como la analgesia, durante todo procedimiento y recuperación. La analgesia suplementaria fue mínima y el porcentaje de fallo fue menor del $9 \%$. L a gran estabilidad hemodinámica, la técnica sencilla y la alta tasa de efectividad hacen del bloqueo femoral tres en uno, una excelente opción para pacientes ASA III y IV con fractura transtocantérica de cadera, que serán sometidos a cirugía.

Palabras claves: Bloque femoral 3 en 1, bloqueo nervio femoral, bloqueo nervio obturador, bloqueo nervio femorocutáneo lateral.

Recibido: 14 de agosto del 2002.

Aceptado: 23 de abril del 2002.

\section{Introducción}

El bloqueo femoral tres en uno, es poco usado en nuestro medio para la cirugía y analgesia del miembro inferior. Este estudio determinará la eficacia del bloqueo femoral tres en uno, para la cirugía de fractura transtrocantérica de cadera, en pacientes ASA III y ASA IV. También se valorará la analgesia postquirúrgica del bloqueo femoral tres en uno.

Este bloqueo se respalda en la idea de la difusión del anestésico local dentro de un espacio que se localiza entre dos capas de fascia, hasta alcanzar el plexo lumbar, que se encuentra limitado en la parte posterior del músculo psoas mayor, y anterior a los procesos transversos lumbares, con el

Especialista en Anestesiología, Hospital Dr. Tomás Casas Casajus. Ciudad Cortés.

2 Coordinador de Docencia. A nestesiología Hospital M éxico. Profesor Universidad de Costa Rica. músculo cuadrado lumbar y el músculo iliaco como pared ${ }^{14}$ (Figura No. 1). El plexo se ubica dentro de una vaina de tejido conectivo, la cual se puede acceder a nivel del ligamento inguinal, lugar donde el nervio femoral entra al muslo( Figura No. 2 ). El anestésico aquí inyectado difunde cefálicamente de este espacio virtual, alcanzando así el plexo lumbar.

El plexo en su disposición usual se ilustra en la Figura No. 3. La primera rama lumbar está unida a la doceava rama torácica; se bifurcan, y la ramificación superior, que es la más larga, se divide para formar los nervios ilioinguinal e iliohi pogástrico; la división inferior se une a la segunda rama lumbar para formar el nervio genitofemoral. La parte restante de la segunda rama lumbar, más la tercera rama lumbar y parte de la cuarta rama lumbar, se unen y luego se subdividen en una porción ventral y otra dorsal.

L as ramas ventrales de la segunda a la cuarta raíz forman el nervio obturador. Las ramas dorsales de la segunda a la tercera se subdividen cada una en otras más pequeñas; la

62 AMC, abril-junio 2002, vol 44 (2) 

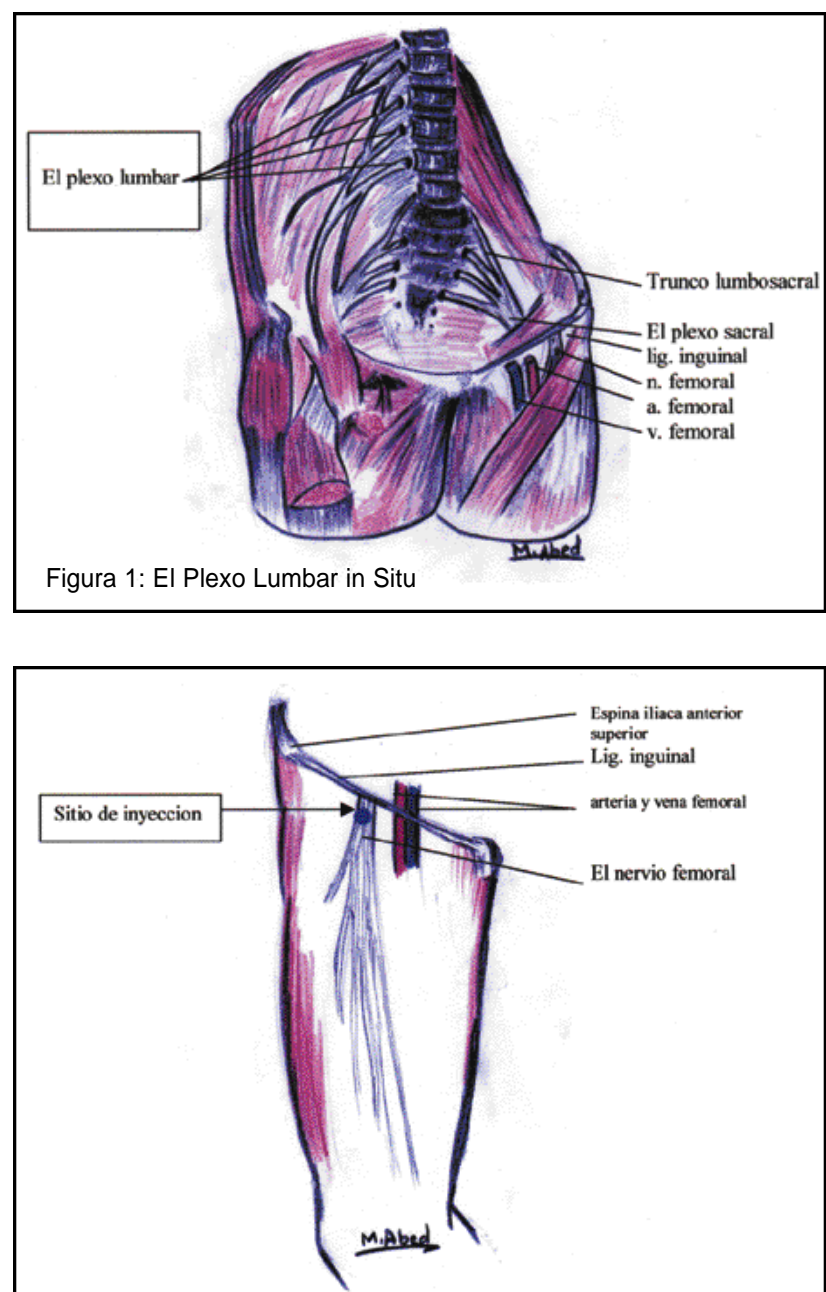

Figura 2: El nervio femoral. Sitio de colocación del bloqueo.

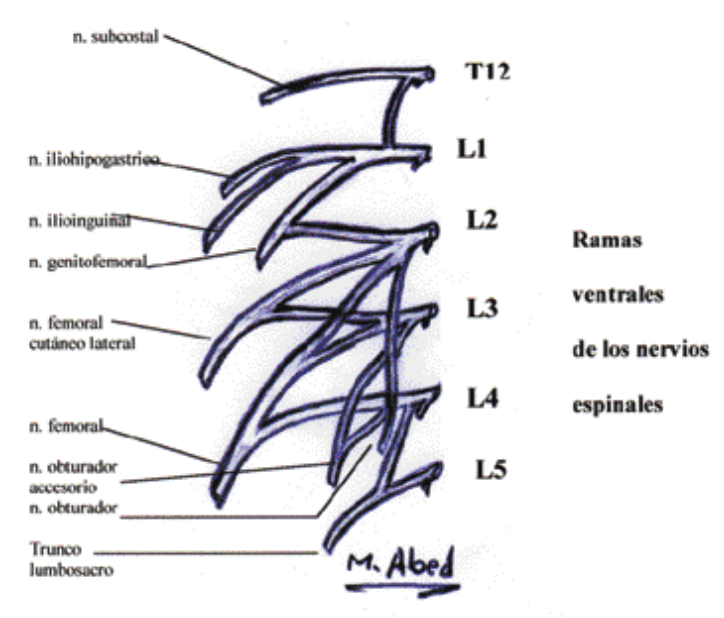

Figura 3: Esquema del Plexo Lumbar menor da lugar al nervio femoral lateral cutáneo; la más grande, unida a la porción dorsal de la cuarta raíz, da origen al nervio femoral.

El nervio obturador accesorio, cuando esté presente, nace de la porción ventral de la tercera y cuarta raíces lumbares.

EI nervio femoral lateral cutáneo emerge del borde lateral del músculo psoas mayor y cruza el iliaco de forma oblicua adelante a la espina ilíaca antero superior. Pasa por detrás 0 atraviesa el ligamento inguinal, variablemente medial a la espina ilíaca antero superior, al rededor de a un centímetro, y anterior 0 atravesando el músculo sartorio en el muslo, donde se divide en una rama anterior y otra posterior. La rama anterior va superficial a unos diez centímetros distales de la espina ilíaca antero superior, supliendo la inervación de la piel de la cara anterior y lateral del muslo y parte de la rodilla. Se une en su porción terminal con la división anterior del nervio femoral y la rama infrapatelar del nervio safeno, formando el plexo patelar. La rama posterior alcanza la fascia lata más arriba que la anterior; se divide e inerva la piel de la cara lateral desde el trocánter mayor hasta la mitad del muslo; también puede suplir la piel del glúteo.

El nervio femoral es la rama más grande del plexo lumbar. Desciende a través del psoas mayor, emerge abajo sobre su borde lateral y pasa a través del psoas y el iliaco, profundo en la fascia iliaca; pasa por detrás del ligamento inguinal y entra al muslo, y se separa en sus divisiones anterior y posterior. Detrás del ligamento inguinal es separado de la arteria femoral por psoas mayor.

Las ramas vasculares de la división posterior del nervio femoral suplen al músculo cuadrado femoral. Una rama al recto femoral entra posterior a su superficie posterior, e inerva la articulación de la cadera

La figura número 4 muestra los dermatomas del miembro inferior.

\section{Materiales y métodos}

El estudio es de tipo prospectivo y se realizó en el Hospital M éxico, entre febrero y agosto de 1998. La investigación fue autorizada por la Coordinación de Postgrado en Anestesiología y Recuperación, y bajo la tutela de los asistentes del servicio, con la supervisión del Dr. M ohammand A bed.

Se seleccionaron doce pacientes programados para cirugía por fractura transtrocantérica de cadera, catalogados como ASA III y ASA IV, según la Sociedad A mericana de A nestesiólogos.

Todos los pacientes fueron monitoreados al momento de su llegada a la sala de operaciones. Se realizaron las mediciones de la tensión arterial no invasiva, con tensiómetros automáticos, y se controló la saturación de oxígeno y monitoreo del ritmo cardiaco, con electrocardiograma continuo en la derivación D II. 
Se instauró un acceso venoso en el miembro superior con catéter $\mathrm{N} 0.18$ en todos los pacientes. Se utilizó solución fisiológica para suplir las demandas de ayuno y el sangrado transoperatorio. En todos los casos se suministro oxígeno a 2 litros por minuto. Se premedicaron con midazolam 3 miligramos, por vía intravenosa, en la sala de operaciones.

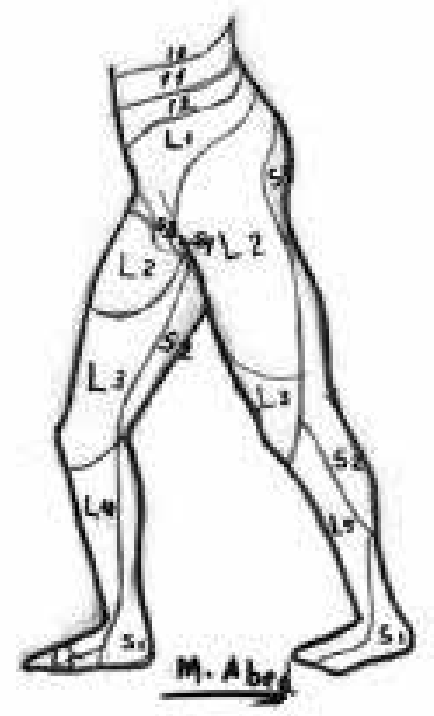

Figura 4: Dermatomas del miembro inferior
El dolor fue medido con puntaje de cero a tres, donde cero equivale a ausencia de dolor, uno a dolor leve, dos a dolor moderado y tres a dolor muy intenso. Se realizaron mediciones en diferentes tiempos, según se observa en el Cuadro Número 4.

Para la realización del bloqueo femoral tres en uno ( Figura Número 2 ) se coloca al paciente en decúbito dorsal, se traza una línea entre la espina ilíaca antero superior y la espina del pubis y se identifica el ligamento inguinal. Se localiza la arteria femoral, aproximadamente 1.5 - 2.5 centímetros laterales al pulso arterial y un centímetro por debajo del ligamento inguinal, en un ángulo de 60 grados con respecto a la piel14 y se introduce una aguja No. 22 X 4 centímetros de largo. Puede aparecer parestesia o no en el nivel de la patela al momento de la punción. Para el bloqueo del nervio fémoro cutáneo lateral se localiza la espina iliaca antero superior, se posiciona la aguja un centímetro medial, se introduce un centímetro y se infiltra la solución anestésica. No se utiliza el estimulador de nervio periférico.

Se utilizaron: 300 miligramos de lidocaína al $2 \%$, más 25 miligramos de bupivacaina al $0.25 \%$; se llevaron a un volumen de $40 \mathrm{cc}$ con solución fisiológica al $0.9 \%$. Se aplicaron 35 centímetros cúbicos en el nivel femoral y cinco centímetros cúbicos para el bloqueo femoral lateral cutáneo ipsilateral.

\section{Resultados}

Se realizaron 12 bloqueos femorales tres en uno, de los cuales siete se dieron en pacientes masculinos y cinco en femeninos (Cuadro No. 2).

La edad promedio fue de 68.3 años, con límite inferior de 37 y superior de 93 años.

64 AMC, abril-junio 2002, vol 44 (2)
Los diagnósticos asociados se especifican en el Cuadro $\mathrm{No}$. 1 y el Gráfico No. 1.

A todos los pacientes, excepto al diagnosticado con encefalopatía, se les aplicó midazolam, 3 miligramos intravenosos al momento de llegar a la sala de operaciones.

Las cifras de los signos vitales registrados en los periodos preoperatorio, transoperatorio y postoperatorio se encuentran en el Cuadro No. 3.

No se presentaron cambios electrocardiográficos con respecto al ritmo basal, en ninguno de los pacientes.

Tres de los pacientes requirieron una dosis de fentanilo 50 microgramos por vía intravenosa, al momento de colocar el soporte perineal en la mesa de tracción. El registro de la percepción del dolor se anota en el Cuadro No. 4.

Un paciente requirió la administración de ketamina $1 \mathrm{mg} / \mathrm{Kg}$ / I.V, ya que no se estableció el bloqueo femoral tres en uno, sin embargo, el bloqueo fémoro cutáneo lateral se estableció parcialmente.

Todos los pacientes mantuvieron saturaciones de oxígeno arriba del $93 \%$.

A todos los pacientes, se le aplicó diclofenaco $1 \mathrm{M} \mathrm{G} / \mathrm{Kg}$ / I.M al momento de su ingreso a la Unidad de Cuidados Posanestésicos.

\section{Discusión}

La eficacia obtenida del bloqueo femoral tres en uno en este estudio en pacientes ASA III y ASA IV es muy alta ( $92 \%$ ), también así lo han demostrado otros estudios ${ }^{6.15}$. La técnica de aplicación, sencilla y rápida ${ }^{10}$, permite que el

\begin{tabular}{|lc|}
\hline \multicolumn{2}{|c|}{$\begin{array}{c}\text { Cuadro No. 1. } \\
\text { Patologías asociadas }\end{array}$} \\
\hline Patología & NUMERO DE CASOS \\
\hline Hipertensión arterial & 5 \\
Hipertensión arterial y diabetes mellitus & 1 \\
Hipertensión arterial y Asma. & 2 \\
Diabetes mellitus y asma & 1 \\
Diabetes mellitus y cardiopatia isquémica & 1 \\
Cardiopatia isquémica y disrritmias & 1 \\
Encefalopatia & 1 \\
\hline TOTAL & 12 \\
\hline $\begin{array}{l}\text { Fuente : Protocolo de recolección de datos bloqueo femoral tres en } \\
\text { uno. Hospital México, San José, Costa Rica. }\end{array}$ \\
Dr. Walter E. Rojas Rivera.
\end{tabular}




\begin{tabular}{|c|c|c|c|}
\hline \multicolumn{4}{|c|}{$\begin{array}{c}\text { Cuadro No.2 } \\
\text { Edad, género y diagnóstico de los pacientes }\end{array}$} \\
\hline Edad & Género & Diagnóstico & Cirugía \\
\hline 41 & Masc. & FxTT & PP \\
\hline 66 & Fem. & FXTT & PP \\
\hline 70 & Masc. & FxTT & PP \\
\hline 89 & Masc. & FXTT & PP \\
\hline 93 & Fem. & FXTT & PP \\
\hline 85 & Fem. & FXTT & PP \\
\hline 62 & Masc. & FXTT & PP \\
\hline 71 & Masc. & FXTT & PP \\
\hline 73 & Masc. & FXTT & PP \\
\hline 37 & Masc. & FXTT & PP \\
\hline 56 & Fem. & FxTT & PP \\
\hline 77 & Fem. & FxTT & PP \\
\hline \multicolumn{4}{|c|}{$\begin{array}{l}\text { Simbología: FXTT= Fractura transtrocantérica de cadera. } \\
\qquad \text { PP= Osteosíntesis con pin placa } \\
\text { Fuente: Protocolo de recolección de datos bloqueo femoral tres en uno. } \\
\text { Hospital México, San José Costa Rica. } 1998 \text {. } \\
\text { Dr. Walter E. Rojas Rivera. }\end{array}$} \\
\hline
\end{tabular}

porcentaje de fallo sea sumamente bajo, aún sin utilizar estimulador de nervio periférico; en un alto porcentaje (92\%) el bloqueo del nervio femoral se lleva a cabo sin el estimulador, versus un $93 \%$ que si lo utiliza; por otra parte, se logra bloquear el nervio fémoro cutáneo lateral hasta en un $41 \%$, sin utilizar el estimulador, contra un $44 \%$ cuando se emplea. Finalmente, el nervio obturador se bloquea en un $68 \%$ y un $78 \%$, sin utilizar y utilizando el estimulador, respectivamente ${ }^{7.15}$. Esto motivó que no se utilizara el estimulador de nervio periférico en el estudio. Del mismo modo, que no se empleara, para aumentar el porcentaje de efectividad de asocio, el bloqueo fémoro cutáneo lateral como refuerzo, dado el bajo porcentaje de bloqueo de este por vía femoral.

En el estudio predominó la población geriátrica con enfermedades sistémicas asociadas, complicadas con fracturas de cadera, patología particularmente frecuente en este grupo etáreo, para el cual varios autores recomiendan tal bloqueo ${ }^{3.6 .7}$ como una alternativa.

La extraordinaria estabilidad hemodinámica observada durante el bloqueo, asociada con una adecuada analgesia, hace pensar que este bloqueo produce cambios mínimos en el nivel simpático, lo que permite manejar a los individuos con menores cargas de líquidos que las requeridas con los bloqueos espinales o peridurales $7 \cdot 10.14$.

Los requerimientos de analgesia suplementaria en el transquirúrgico pueden ser necesarios por fallas técnicas, tales como el desplazamiento involuntario de la aguja durante la inyección del anestésico, 0 por factores anatómicos ${ }^{13}$. que predisponen a un ascenso tórpido del anestésico por la vaína aponeurótica, lo que se refleja como un bloqueo mal instaurado, o bien, en parches. Sin embargo, las dosis coadyuvantes, de anestésicos por vía intravenosa, necesarias para complementar la anestesia con este tipo de bloqueo, son significativamente inferiores 9 .

En lo que se refiere a la anal gesia postquirúrgica, se observó una marcada disminución en los requerimientos de

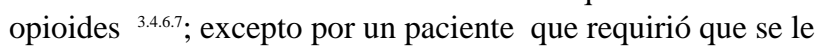
administrara tramadol en la Unidad de Recuperación, el resto se manejó adecuadamente con dicl ofenaco a 1 M G / K g/ I.M. Se utilizó este AINE con el propósito de aprovechar su acción antiinflamatoria y mantener niveles séricos del fármaco, para conservar la analgesia en el postquirúrgico.

\begin{tabular}{|c|c|c|c|c|c|c|c|c|c|c|c|c|c|c|c|}
\hline \multicolumn{16}{|c|}{$\begin{array}{c}\text { Cuadro No.3 } \\
\text { Registro de Signos Vitales de los Pacientes }\end{array}$} \\
\hline & \multicolumn{3}{|c|}{ Prebloqueo } & \multicolumn{3}{|c|}{10 minutos } & \multicolumn{3}{|c|}{ Incisión } & \multicolumn{3}{|c|}{ Óseo } & \multicolumn{3}{|c|}{ Recuperación } \\
\hline & PAS & PAD & FC & PAS & PAD & FC & PAS & PAD & FC & PAS & PAD & FC & PAS & PAD & FC \\
\hline 1 & 150 & 90 & 90 & 155 & 85 & 80 & 150 & 90 & 85 & 150 & 90 & 85 & 160 & 90 & 85 \\
\hline 2 & 160 & 80 & 60 & 160 & 90 & 60 & 160 & 90 & 70 & 160 & 85 & 70 & 160 & 85 & 70 \\
\hline 3 & 150 & 90 & 75 & 150 & 90 & 80 & 155 & 85 & 75 & 150 & 80 & 75 & 150 & 90 & 75 \\
\hline 4 & 180 & 95 & 80 & 180 & 90 & 75 & 175 & 85 & 80 & 180 & 90 & 80 & 180 & 95 & 75 \\
\hline 5 & 190 & 100 & 86 & 175 & 90 & 90 & 170 & 90 & 85 & 170 & 90 & 80 & 170 & 85 & 80 \\
\hline 6 & 180 & 100 & 76 & 160 & 90 & 80 & 160 & 75 & 80 & 170 & 60 & 85 & 170 & 70 & 80 \\
\hline 7 & 160 & 70 & 85 & 160 & 70 & 85 & 160 & 80 & 80 & 165 & 80 & 80 & 165 & 90 & 80 \\
\hline 8 & 170 & 90 & 80 & 165 & 90 & 85 & 160 & 90 & 80 & 150 & 80 & 80 & 160 & 80 & 80 \\
\hline 9 & 170 & 100 & 85 & 180 & 90 & 80 & 190 & 100 & 110 & 180 & 95 & 100 & 180 & 90 & 100 \\
\hline 10 & 140 & 90 & 90 & 130 & 80 & 90 & 135 & 80 & 90 & 130 & 70 & 85 & 130 & 80 & 85 \\
\hline 11 & 160 & 90 & 95 & 160 & 95 & 90 & 155 & 90 & 85 & 160 & 80 & 80 & 160 & 90 & 85 \\
\hline 12 & 185 & 95 & 60 & 180 & 95 & 55 & 175 & 70 & 60 & 170 & 80 & 60 & 170 & 90 & 60 \\
\hline & plogía: & $\begin{array}{l}P A S=P r \\
P A D=P r \\
F C=F r\end{array}$ & & $\begin{array}{l}\text { diast } \\
\text { diaca }\end{array}$ & & 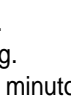 & & & & $\begin{array}{l}\text { D. San } \\
\text { ojas R }\end{array}$ & sélec & $\begin{array}{l}\text { de de } \\
\text { Zica. }\end{array}$ & & & s en uno \\
\hline
\end{tabular}




\begin{tabular}{|c|c|c|c|c|c|c|c|c|c|}
\hline \multicolumn{10}{|c|}{$\begin{array}{c}\text { Cuadro No. } 4 \\
\text { Registro de percepción de dolor y } \\
\text { Anestesia suplementaria }\end{array}$} \\
\hline \multicolumn{2}{|r|}{ Prebloqueo } & \multicolumn{2}{|c|}{10 minutos } & \multicolumn{2}{|c|}{ Incisión } & \multicolumn{2}{|c|}{ Óseo } & \multicolumn{2}{|c|}{ Recuperación } \\
\hline & Dolo A.S & Dolo & A.S & Dolor & A.S & Dolor & A.S & Dolor & A.S \\
\hline 1 & 2 & 0 & & 0 & & 0 & & 0 & $\begin{array}{l}\text { Diclofenaco } \\
1 \mathrm{mg} / \mathrm{kg} / \mathrm{MM}\end{array}$ \\
\hline 2 & 3 & 0 & & 0 & & 0 & & 0 & $\begin{array}{l}\text { Diclofenaco } \\
1 \mathrm{mg} / \mathrm{kg} / \mathrm{IM}\end{array}$ \\
\hline 3 & 2 & 1 & $\begin{array}{l}\text { Fentanil } \\
50 \mathrm{mg}\end{array}$ & 0 & & 0 & & 0 & $\begin{array}{l}\text { Diclofenaco } \\
1 \mathrm{mg} / \mathrm{kg} / \mathrm{IM}\end{array}$ \\
\hline 4 & 3 & 0 & & 0 & & 0 & & 0 & $\begin{array}{l}\text { Diclofenaco } \\
1 \mathrm{mg} / \mathrm{kg} / \mathrm{MM}\end{array}$ \\
\hline 5 & 2 & 0 & & 0 & & 0 & & 0 & $\begin{array}{l}\text { Diclofenaco } \\
1 \mathrm{mg} / \mathrm{kg} / \mathrm{IM}\end{array}$ \\
\hline 6 & 2 & 0 & & 0 & & 0 & & 0 & $\begin{array}{l}\text { Diclofenaco } \\
1 \mathrm{mg} / \mathrm{kg} / \mathrm{M}\end{array}$ \\
\hline 7 & 2 & 1 & $\begin{array}{l}\text { Fentanil } \\
50 \mathrm{mg}\end{array}$ & 0 & & 0 & & 0 & $\begin{array}{l}\text { Diclofenaco } \\
1 \mathrm{mg} / \mathrm{kg} / \mathrm{M}\end{array}$ \\
\hline 8 & 2 & 0 & & 0 & & 0 & & 0 & $\begin{array}{l}\text { Diclofenaco } \\
1 \mathrm{mg} / \mathrm{kg} / \mathrm{lM}\end{array}$ \\
\hline 9 & 3 & 3 & $\begin{array}{l}\text { Ketamina } \\
100 \mathrm{mg}\end{array}$ & 1 & $\begin{array}{l}\text { Ketamina } \\
50 \mathrm{mg}\end{array}$ & 1 & $\begin{array}{l}\text { Ketamina } \\
40 \mathrm{mg}\end{array}$ & 1 & $\begin{array}{l}\text { Diclofenaco } \\
1 \mathrm{mg} / \mathrm{kg} / \mathrm{IM}\end{array}$ \\
\hline 10 & 3 & 0 & & 0 & & 0 & & 0 & $\begin{array}{l}\text { Diclofenaco } \\
1 \mathrm{mg} / \mathrm{kg} / \mathrm{M}\end{array}$ \\
\hline 11 & 2 & 0 & & 0 & & 0 & & 0 & $\begin{array}{l}\text { Diclofenaco } \\
1 \mathrm{mg} / \mathrm{kg} / \mathrm{M}\end{array}$ \\
\hline 12 & 3 & 1 & $\begin{array}{l}\text { Fentanil } \\
50 \mathrm{mg} \\
\text { DHBP2.5 mg }\end{array}$ & 0 & & 0 & & 0 & $\begin{array}{l}\text { Diclofenaco } \\
1 \mathrm{mg} / \mathrm{kg} / \mathrm{lM}\end{array}$ \\
\hline
\end{tabular}

Simbología: A.S = Anestesia Suplementaria $\quad \mathrm{DHBP}=$ Dehidrobenzoperidol

Escala del dolor: $0=$ No dolor. $1=$ Dolor leve. $2=$ Dolor Moderado. $3=$ Dolor severo .

Fuente: Protocolo de recolección de datos bloqueo femoral tres en uno.

Hospital México, San José Costa Rica. 1998.

Dr. Walter E. Rojas Rivera. informado algunos casos de parálisis motora prolongada con el uso de bupivacaína ${ }^{9}$, sin embargo, aún cuando se sobrepase en un $50 \%$ la dosis máxima recomendada por el fabricante, no se alcanzaron valores pico en el nivel plasmático, según el estudio de Misra y colaboradores ${ }^{11}$. Varias investigaciones sugieren que el volumen por emplear está entre los 20 y 40 centímetros cúbicos ${ }^{2.5 .10 .14 .15}$, dado que dentro de este rango no varía la extensión del bloqueo, y los cambios en la concentración del anestésico no tienen mayor relevancia en el resultado final del bloqueo $0^{10.15 .16}$. También es recomendable el uso de bicarbonato de sodio para alcalinizar la solución y lograr un tiempo de latencia menor ${ }^{2}$.

Se postulan otras variantes de la técnica del bloqueo femoral tres en uno, como lo es el bloqueo continuo con catéter, asociado con una bomba para P.C.A. (A nalgesia Controlada por el Paciente), que ofrece un periodo mayor de analgesia, con la ventaja de ser regulada por el mismo individuo y facilitar la realización de técnicas quirúrgicas más prolongadas ${ }^{5.16}$.

Desde el punto de vista de costos, este bloqueo es una opción para aquellos pacientes en los cuales se posponía el acto quirúrgico por su labilidad metabólica 0 hemodinámica, disminuyendo la estancia hospitalaria y las complicaciones por encamamiento prolongado. A demás, hay una disminución de los requerimientos farmacológicos para la analgesia postoperatoria.
Por otra parte, se perfila como un complemento para aquellos pacientes que han recibido otro tipo de anestesia, ya sea neuroaxial o general, en los que se desea una analgesia postquirúrgica prolongada, 0 bien disminuir los requerimientos transanestésicos en la anestesia general.

L a combinación de anestésicos utilizada permite un bloqueo de instauración rápida por efecto de la lidocaína y un efecto prolongado logrado por la bupivacaína, que según varios estudios posibilita una analgesia postquirúrgica de entre 24 y 48 horas $^{4.67 .10}$, sin necesidad de anal gesia suplementaria, 0 con requerimientos mínimos. Si bien estas son las drogas anestésicas disponibles en nuestro medio, se determina que para el bloqueo femoral tres en uno, el anestésico local que ofrece mejores resultados es la mepivacaína².

Con la concentración y dosis utilizadas, no se presentaron complicaciones metabólicas o neurológicas ${ }^{7}$, aunque se han

\section{Conclusiones}

Los resultados obtenidos con la utilización de este bloqueo, en concordancia con múltiples estudios sobre el tema, respaldan la eficacia del bloqueo femoral tres en uno para la cirugía de cadera. Esta técnica por su fácil aplicación, alto porcentaje de efectividad y la gran estabilidad hemodinámica que exhibe, se postula como el bloqueo de elección en los pacientes A SA III y A SA IV con fractura transtrocantérica de cadera, en los que por su condición, la homeostasis se encuentra en un frágil equilibrio, que puede ser alterado por las técnicas de bloqueo del neuroeje, las cuales implican cambios hemodinámicos importantes que aún con una adecuada hidratación no son del todo prevenibles, o bien se puede llevar al paciente a una sobrecarga de volumen con todas las implicaciones que esto conlleva.

Por otra parte la excelente analgesia postoperatoria que se obtiene, que se prolonga 48 horas, lo convierten en un 
bloqueo ideal para estos pacientes y por otra parte se perfila como un complemento para aquellos pacientes que han recibido otro tipo de anestesia, ya sea regional o general, en los que se desea una analgesia posquirúrgica prolongada 0 bien para disminuir los requerimientos transanestésicos en la anestesia general.

\section{Abstract}

Twelve three in one femoral blocks were done for osteosynthesis with pin plate in transtrochanteric hip fracture in patients ASA III and ASA IV. It was used bupivacain $0,25 \%$ and lidocain $2 \%$ to a $40 \mathrm{ml}$ volume. Observed hemodynamic stability was excellent, thus analgesic during the procedure and recover. Supplementary analgesic was minimal and the fail rate was less than $9 \%$.

Great hemodynamic stability, the simplicity of the procedure and the high efectiveness rate, made of the three in one femoral block an excellent choice for patients ASA III and ASA IV with transtrochanteric hip fracture that will need surgery.

\section{Key words}

Three in one femoral block. Femoral nerve block. Obturator nerve block. Lateral femorocutaneous nerve block.

\section{Referencias}

1. Bonicalzi, V. Gallino, M. Comparation of two regional anesthesic techniques for knee artroscopy. A rthroscopy. 1995. A pr; 11(2): 207-12.

2. Capogna, G. et al. Alkalinization of local anesthesics. Wich block, wich local anesthesic ?. Reg-A nesth. 1995. Sep-Oct; 20(5): 369-72.

3. Coad, NR. Post-operative anal gesia following femoral neck surgery a comparison betwen 3 - in -1 femoral nerve block and lateral cutaneus nerve block. Eur-J-A naesthesiol. $1991 \mathrm{~J} \mathrm{ul;} 8$ (4): 287-90.

4. Edkin, BS. Et al. Femoral nerve block as an alternative to parenteral narcotics for pain control after anterior cruciate ligament reconstruction. A rthroscopy. 1995. Aug; 11 (4): 404-9.

5. Edwards, ND. Wrigth, EM. Continuos low dose 3 - in - 1 nerve blockade for postoperative pain relief after total knee replacement. A nesth-A nal g. 1992. A ug; 75 (2): 265-7.

6. Haddad, FS. Williams, RI. Femoral nerve block in extracapsular femoral neck fractures. J-B one-J oint-Surg. 1995. N ov; 77(6):922-3.

7. Hood, G. et al. Postoperative analgesia after triple nerve block for fractured neck of femur. A naesthesia. 1991. Feb; 46 (2): 138-40.

8. Lang, SA. Et al. The femoral 3 - in - 1 block revisited. J-Clin-A nesth. 1993. Jul-A ug; 5(4): 292-6.

9. Lynch,J. Prolongeg motor weakness after femoral nerve block with bupivacaine $0.5 \%$. A naesthesia. 1990. M ay; 45 (5): 421.

10. Miller, Ronald. A nestesia. 1993. Ediciones Doyma. M éxico. pp: 1289 - 1292.

11. Misra, U. et al. Plasma concentrations of bupivacaine following combined sciatic and femoral 3 in 1 nerve blocks in open knee surgery. Br-J-A naesth. $1991 \mathrm{M}$ ar; 66 (3): 310 - 3.
12. Netter, F. Interactive A tlas of Human A natomy. Ciba Geigy M edical Education and Publications. USA. 1995

13. Ritter, JW. Femoral nerve "sheath" for inguinal paravascular lumbar plexus block is not found in human cadavers. J-Clin-A nesth. 1995. Sep; 7 (6): 470-3.

14. Scott, D. Bruce. et al. Técnicas de anestesia regional. 1989. Editorial M édica Panamericana. España. pp: 122 - 127.

15. Seeberguer, M D. U rwyler, A. Paravascular lumbar plexus block: block extension after femoral nerve stimulation and inyection of $20 \mathrm{vs} .40 \mathrm{ml}$ mepivacaina 10 mg/ml. A cta-A naesthesiol-Scand. 1995. A ug; 39 (6): 763-73.

16. Tetzlaff, JE. et al. Effectiveness of bupivacaine administred via femoral nerve catheter for pain control after anterior cruciate ligament repair. J-Clin-A nesth. 1997. Nov; 9 (7): 542-5.

17. Williams, Peter. et al. Gray"s A natomy. 1989. Churchill Livingstone. 37aㅡ ed. United Kingdom. pp: 635 - 640, 1140 - 1144, 1152 - 1154. 